\title{
A periodic approach for input-delay problems: application to network controlled systems affected by polytopic uncertainties
}

\author{
Jean-François Trégouët ${ }^{1 *}$, Alexandre Seuret ${ }^{2}$ and Michaël Di Loreto ${ }^{1}$ \\ ${ }^{1}$ Université de Lyon, INSA-Lyon, Laboratoire Ampère CNRS UMR 5005, 20 Avenue Albert Einstein, F-69621, \\ Villeurbanne, France \\ ${ }^{2}$ CNRS, LAAS, 7 avenue du colonel Roche, F-31400 Toulouse, France and Univ de Toulouse, LAAS, F-31400 Toulouse, \\ France
}

\begin{abstract}
SUMMARY
This paper deals with robust stability and stabilization of linear discrete-time systems subject to uncertainties and network constraints. In network control systems, the control loop is closed over a network, which induces additional dynamics to the original control loop such as delays, sampling, quantization among many others. This paper focuses on networked induced delays due to unreliable network for which packet losses may occur. An equivalent periodic-like representation of the resulting system is proposed. This allows first, to revisit existing results in this framework and second, to take model uncertainties into account by analyzing the closed-loop model by means of a recent method based on robust control for discrete-time time-varying systems. Stability analysis and dynamic state-feedback stabilization are characterized via new conditions, whose conservatism is extensively discussed. Effectiveness of the proposed methodology is illustrated by numerical examples. Copyright (c) 0000 John Wiley \& Sons, Ltd.
\end{abstract}

Received ...

KEY WORDS: Discrete-time systems, delays, packet losses, robust analysis, stability, stabilization

\section{INTRODUCTION}

Due to the emergence of new technologies, it is now reasonable to consider applications where the various components of a control loop are closed through a single or several wireless network links. The benefits of such a distribution of the components are for instance the possibility to avoid closing the loop with wires, to get scalability properties among many others. The network may induce additional dynamics, which may disturb or even destroy the performances and stability properties of the closed-loop system. Among these dynamics, one of the most studied in the context of networked control systems is due to packet losses [1,2]. This phenomenon appears when a (wireless) network link is not reliable and data packets are often lost between transmitters and receivers. In some applications such as in underwater communication or multi-hop networks, analyzing the effect

$\overline{{ }^{*} \text { Correspondence }}$ to: E-mail: jean-francois.tregouet@insa-lyon.fr

Copyright (C) 0000 John Wiley \& Sons, Ltd.

Prepared using rncauth.cls [Version: 2010/03/27 v2.00] 
of this phenomenon in a control loop becomes crucial. It is usually assumed that, when a data packet is lost, the control input will use the last available data to compute the next control input. Among the possible solutions to address this problem, one may look at a method based on an equivalent formulation of the input delay approach for continuous-time sampled-data systems [3] to discrete-time systems [4]. The interest of this method is that the network closed-loop system subject to packet losses is rewritten as a system affected by a time-varying input delay governed by a particular dynamic. In the context of the stability analysis of discrete-time delay systems, several methods have been provided in the literature to derive delay-dependent stability conditions. The reader may refer for instance to [5, 6, 7, 8, 9] for Lyapunov-Krasovskii methods, to [10] for Integral Quadratic Constraints approaches, or to [11, 12] for switched systems approaches with average dwell times conditions [13]. Notably, an equivalence between the Lyapunov-Krasovskii and the switched systems approaches was shown in [14].

Most of these papers consider that the time-varying delay function is bounded by some known and constant lower and upper bounds. Therefore, since no assumptions are required on the variations of the delay function, these papers are concerned with a more general class of systems with time-varying delays than control systems with packet losses. In order to have a more dedicated analysis, several approaches have been considered. In [15], the problem is recast into a finite dimensional discrete-time jump linear system with transition jumps being modeled as finite-state Markov processes. In [10], a particular attention has been paid to include additional assumptions on the variations of the delay function. In $[16,12,17]$, methods to take into account in an exact manner the packet dropout phenomena are proposed. A switched system approach is used which leads to sufficient stability conditions.

In this paper, we focus on control system subject not only to packet losses but also to polytopictype model uncertainties. In such a case, the stability analysis strategy proposed in [17] comes up with Linear Matrix Inequality (LMI) conditions which are non linear with respect to the system matrices, preventing then from an extension to robust stability. In the present paper, this difficulty is tackled by constructing an equivalent time-varying augmented model from the original closed-loop systems with time-delay. It appears that the obtained time-varying model switches from a periodic dynamics to another. The roots of this approach can be traced back to $[18,19]$, where periodic controllers with memory were considered, and to [20], which deals with asynchronous sampling. This reformulation allows (i) to revisit the stability conditions of [17] and, in particular, to establish that when the time-delay is constant, those conditions are also necessary and their associated numerical complexity is independent from the value of the time-delay and (ii) to develop a novel methodology for the analysis and design of such control systems which, in constrast with [17], consider time-varying state-feedback controller gains. The latter achievement relies on a relaxation technique preserving linearity with respect to system matrices. Conservatism associated to new LMI conditions is extensively discussed. Numerical examples illustrate the effectiveness of the proposed methodology as well as the benefits of allowing the controller gains to be time-varying.

The paper is organized as follows. Section 2 describes the problem formulation. Section 3 and 4 develop the analysis and the control design of nominal and uncertain systems, respectively. Some numerical examples are presented in Section 5.

The following notations are used throughout the paper. The symbol $\mathbf{1}_{n}$ stands for the identity matrix of dimensions $n \times n$. The null matrix of size $m \times n$ is denoted by $\mathbf{0}_{m \times n}$. When the 
dimensions are clear from the context, they are omitted. The set of $l \times l$ symmetric matrices (respectively positive-definite symmetric matrices) are denoted by $\mathbb{S}^{l}$ (resp. $\mathbb{S}_{+}^{l}$ ). For a real square matrix $A$, we define $\operatorname{He}\{A\}=A+A^{T}$. For a matrix $R, R^{\perp}$ is such that $R^{\perp} R=\mathbf{0}$. The operator 'diag' builds block-diagonal matrix from input arguments. For given integers $k$ and $N$, we denote by $\lceil k\rceil_{N}$ the remainder of $k$ divided by $N$. The symbol $\star$ is used to complete symmetric matrices.

The following lemma results from standard application of Finsler's Lemma (see e.g. [21]).

\section{Lemma 1}

For any matrix $Q$ and $R$ of appropriate dimensions and such that $Q$ is symmetric, the following matrix inequalities are equivalent:

$$
\begin{aligned}
R^{T \perp} Q R^{T \perp T} & \prec 0 \\
\exists \rho \in \mathbb{R}: Q+\rho R^{T} R & \prec 0 \\
\exists \mathcal{F}: Q+\operatorname{He}\{\mathcal{F} R\} & \prec 0
\end{aligned}
$$

\section{PROBLEM STATEMENT}

Consider the linear discrete-time system

$$
\Sigma(\theta): \quad x_{k+1}=A(\theta) x_{k}+B(\theta) u_{k},
$$

where $x_{k} \in \mathbb{R}^{n}$ and $u_{k} \in \mathbb{R}^{m}$ refer to the state and the input vectors, respectively. This model is subject to polytopic uncertainties such that

$$
\left[\begin{array}{ll}
A(\theta) & B(\theta)
\end{array}\right]=\sum_{q=1}^{L} \theta_{q}\left[\begin{array}{ll}
A^{[q]} & \left.B^{[q]}\right],
\end{array}\right.
$$

with $A^{[q]} \in \mathbb{R}^{n \times n}$ and $B^{[q]} \in \mathbb{R}^{n \times m}$ when the uncertain vector $\theta:=\left(\theta_{1}, \ldots, \theta_{L}\right)$ belongs to the unit simplex $\Theta_{L}$ defined by

$$
\Theta_{L}:=\left\{\left(\theta_{1}, \ldots, \theta_{L}\right) \in[0,1]^{L} \text { s.t. } \theta_{1}+\ldots+\theta_{L}=1\right\} .
$$

Throughout this paper, $\theta$ is assumed to be time-invariant.

Let $\left\{n_{p}\right\}_{p \in \mathbb{N}}$ be an increasing sequence of positive integers such that $\bigcup_{p \in \mathbb{N}}\left[n_{p}, n_{p+1}\right)=[0, \quad+$ $\infty)$ and for which there exists a strictly positive integer $N_{\max }$ such that

$$
\forall p \in \mathbb{N}, \quad N_{p}:=n_{p+1}-n_{p} \in \mathcal{I}:=\left\{1, \ldots, N_{\max }\right\}
$$

The sequence $\left\{n_{p}\right\}_{p \in \mathbb{N}}$ represents the instants where the controller receives new data from the sensors. The control input $u_{k}$ is computed as soon as the information from the sensors arrives and is implemented until a more recent information coming from the sensor is received (see Figure 1). The time interval between the current instant time and the last packet receipt, denoted by $d_{k}$, is given by

$$
d_{k}:=k-n_{p} \geq 0, \forall k \in\left[n_{p}, n_{p+1}\right) .
$$




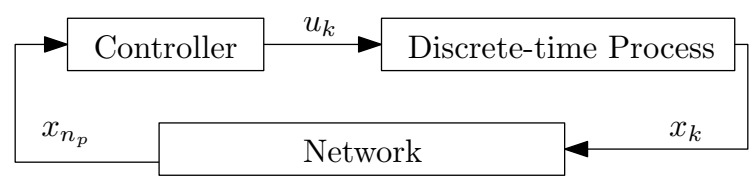

Figure 1. Network control system of interest

The design of the controller is performed for two classes of controllers (time-invariant and timevarying state-feedback), namely

$$
\begin{aligned}
& \forall k \in\left[n_{p}, n_{p+1}\right), \quad u_{k}=K x_{n_{p}}, \\
& \forall k \in\left[n_{p}, n_{p+1}\right), \quad u_{k}=K_{k} x_{n_{p}},
\end{aligned}
$$

where both $K$ and $K_{k}$ belong to $\mathbb{R}^{m \times n}$. Additionally, we assume that the controller gains in (9b) only depend on the value of the time interval $d_{k}$, so that $K_{k}=K_{d_{k}}$ for any $k \in\left[n_{p}, n_{p+1}\right)$. The closed-loop system (4) and (9b) becomes

$$
x_{k+1}=A(\theta) x_{k}+B(\theta) K_{k} x_{n_{p}}, \forall k \in\left[n_{p}, n_{p+1}\right) .
$$

This class of systems can be realized by a time-delay system with time-increasing state delay on each interval $\left[n_{p}, n_{p+1}\right)$, as illustrated in Figure 2. Indeed, since $x_{n_{p}}=x_{k-k+n_{p}}=x_{k-d_{k}}$ and $K_{k}=K_{d_{k}}^{\dagger}$, the system (10) takes the form

$$
\Sigma_{c l}(\theta): x_{k+1}=A(\theta) x_{k}+B(\theta) K_{d_{k}} x_{k-d_{k}}, \forall k \in\left[n_{p}, n_{p+1}\right)
$$

This manipulation refers to the input delay approach proposed in [3] and has been already successfully applied in the context of continuous sampled-data systems for the time-invariant controller (9a). In the context of discrete-time systems, this model transformation has already been employed in [4].

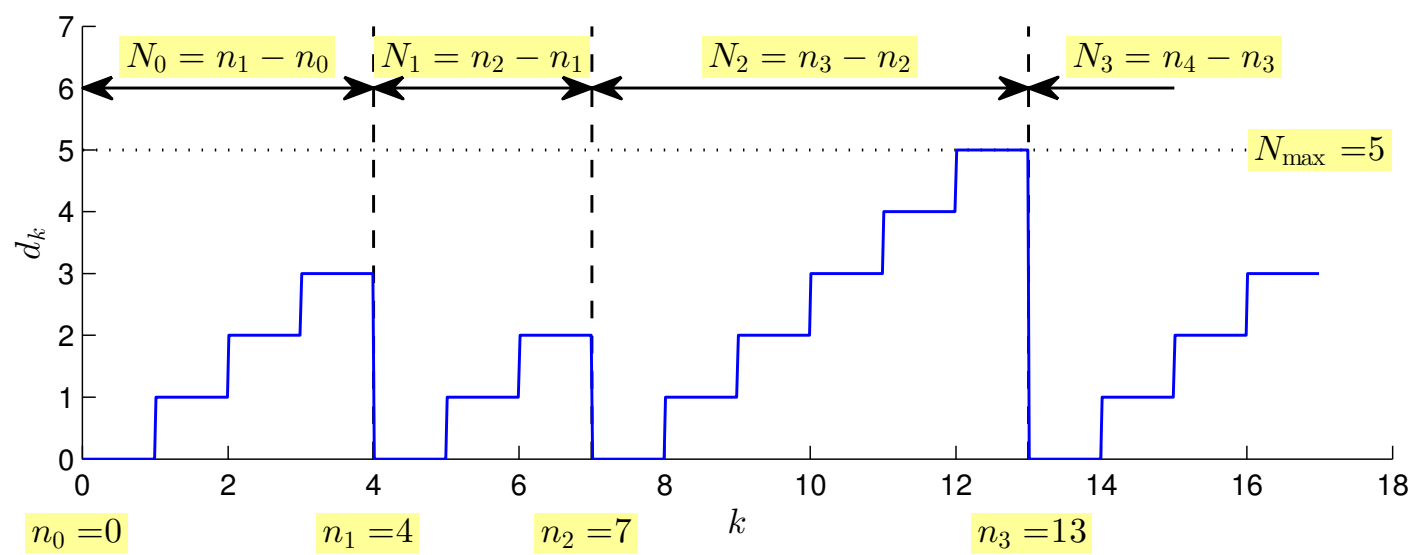

Figure 2. Example of chronograph of $d_{k}$

\footnotetext{
${ }^{\dagger}$ In the packet drop scenario illustrated in Figure 2, controller (9b) with $K_{k}=K_{d_{k}}$ reads $K_{0}=K_{4}=K_{13}=\ldots, K_{1}=$ $K_{5}=K_{8}=K_{14}=\ldots, K_{2}=K_{6}=K_{9}=\ldots$ and so on and so forth. 
In the following, both analysis and synthesis problems are tackled. That is, we provide conditions assessing robust asymptotic stability of $\Sigma_{c l}(\theta)$ and derive conditions leading to control laws (9a) (or (9b)) ensuring that $\Sigma_{c l}(\theta)$ is robustly stable. As a preliminary step, Section 3 deals with the simpler case where $\theta$ is known. Then, the full uncertain problem is addressed in Section 4.

\section{Remark}

The reasons for imposing the constraint $K_{k}=K_{d_{k}}$, and hence focusing our attention on a particular class of time-varying state-feedback, are twofold. It first originates from the intuition of copying the intrinsic time-delay dynamics of the discrete-time process together with the network into the controller so that, in (11), $x_{k+1}$ is a function of $d_{k}$ solely whereas it depends on both $k$ and $d_{k}$ in (10). Second, it gives a way to write the controller gains solving the synthesis problem as the solution of a semi-definite program, as demonstrated afterward. Indeed, $K_{k}=K_{d_{k}}$ implies that there exists a finite number of controller gains $K_{k}$ since $d_{k}$ is assumed to be bounded (see (7) and (8)).

\section{THE NOMINAL PROBLEM WHERE $\theta$ IS KNOWN}

As a preliminary study, let us first assume that $\theta$ is known. Thus, the dependency of $A$ and $B$ with respect to $\theta$ is omitted in this section.

\subsection{An augmented model reformulation}

Using the manipulation introduced in [19], the closed-loop model $\Sigma_{c l}$ is treated via the following state-space formulation where both coefficients and dimensions of the state matrix $\bar{A}_{d_{k}}$ are timevarying:

$$
\bar{x}_{k+1}=\bar{A}_{d_{k}} \bar{x}_{k},
$$

with

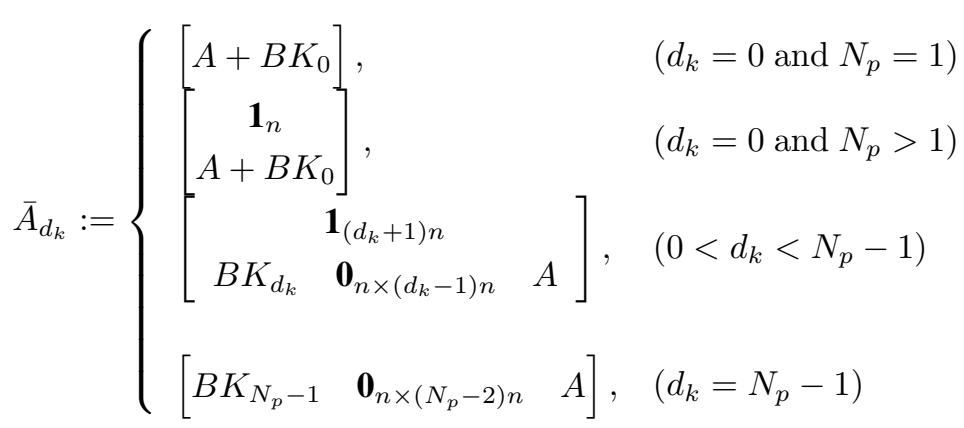

and where the augmented state vector $\bar{x}_{k}$ corresponds to

$$
\forall k \in\left[n_{p}, n_{p+1}\right), \bar{x}_{k}=\left(x_{n_{p}}, \cdots, x_{k}\right) \in \mathbb{R}^{\left(d_{k}+1\right) n} .
$$

Clearly, this reformulation preserves stability, i.e. (11) is stable if and only if (12) is stable. This is the reason why, in this paper, the stability analysis of $\Sigma_{c l}$ is handled by means of (12) which, in contrast with (11), does not have any time-delay dynamics. For this purpose, we introduce the 
state-transition matrix $\Psi_{N_{p}}$ between $x_{n_{p}}=\bar{x}_{n_{p}}$ and $x_{n_{p+1}}=\bar{x}_{n_{p+1}}$ by

$$
\Psi_{N_{p}}:=\prod_{k=0}^{N_{p}-1} \bar{A}_{N_{p}-1-k}=A^{N_{p}}+\sum_{k=0}^{N_{p}-1} A^{N_{p}-1-k} B K_{k} .
$$

\subsection{Regularly information receipts}

As a preliminary step, it is first assumed that the time-interval between two information receipts has a constant value denoted by $N$. This is equivalent to saying that $\mathcal{I}=\{N\}$ where $\mathcal{I}$ is defined by (7). In this case, $d_{k}$ is governed by $d_{k+1}=\left\lceil d_{k}+1\right\rceil_{N}$ which clearly corresponds to a periodic dynamics with period $N$.

For a given controller (9b), consider first the closed-loop stability analysis problem. Relying on the observation that $d_{k}$ is periodic, the following lemma easily derives from the well-known fact that stability of periodic system is equivalent to Schur stability of $\Psi_{N} .^{\ddagger}$

Lemma 2 (Nominal analysis, [22])

If $\mathcal{I}=\{N\}$, the system $\Sigma_{c l}$ is stable if and only if there exists $P \in \mathbb{S}_{+}^{n}$ such that

$$
\Psi_{N}^{T} P \Psi_{N}-P \prec 0
$$

holds.

The matrix $\Psi_{N}$ coincides with the state-transition matrix from $\bar{x}_{n_{p}}=x_{n_{p}}$ to $\bar{x}_{n_{p+1}}=x_{n_{p+1}}$, that is

$$
\bar{x}_{n_{p+1}}=\Psi_{N} \bar{x}_{n_{p}} .
$$

We can also note that condition (16) can be regarded as an asymptotic stability condition of (17), assessed through the strict decrease of the Lyapunov functionnal $V\left(\bar{x}_{n_{p}}\right)=\bar{x}_{n_{p}}^{T} P \bar{x}_{n_{p}}$. Thus, when $\mathcal{I}=\{N\}$, the model (12) (and hence $\Sigma_{c l}$ ) is stable if and only if the application $\bar{x}_{n_{p}} \rightarrow \bar{x}_{n_{p+1}}=$ $\bar{x}_{n_{p}+N}$ is a contraction mapping. Although this fact is well-known from periodic systems theory, this will be of major importance in the sequel when $N_{p}$ will be considered as unknown.

The corresponding stabilization problem, that is finding the gains of the controller (9b) which stabilizes the closed-loop, is now solved by means of Lemma 2.

Lemma 3 (Nominal synthesis)

If $\mathcal{I}=\{N\}$, controller (9b) stabilizes the closed-loop model $\Sigma_{c l}$ if and only if there exist $W \in \mathbb{S}_{+}^{n}$ and $Y_{k} \in \mathbb{R}^{m \times n}$ for all $k \in\{0, \ldots, N-1\}$ such that the following condition holds:

$$
\left[\begin{array}{cc}
-W & \star \\
A^{N} W+\sum_{k=0}^{N-1} A^{N-1-k} B Y_{k} & -W
\end{array}\right] \prec 0 .
$$

The gains of the control law (9b) are obtained by

$$
K_{k}=Y_{k} W^{-1}
$$

${ }_{\ddagger}^{\ddagger}$ In this context, $\Psi_{N}$ corresponds to the so-called monodromy matrix (see e.g. [22]). 
for all $k \in\{0, \ldots, N-1\}$.

Proof

Substituting $Y_{k}$ by $K_{k} W$ in (18) and recognizing the expression of $\Psi_{N}$ given by (15) leads to

$$
\left[\begin{array}{cc}
-W & \star \\
\Psi_{N} W & -W
\end{array}\right] \prec 0,
$$

which can be proved to be equivalent to (16) with $P=W^{-1}$ using a Schur complement. The use of Lemma 2 completes the proof.

\section{Remark}

While the Semi-Definite Program (SDP) defined in Lemma 2 depends on $N$, it is remarkable that neither its number of variables nor its number of rows depend on this parameter $N$. Hence, numerical complexity associated with this lemma is not affected by the time interval between two information receipts. SDP of Lemma 3 is slightly different with that respect since its number of variables depends on $N$ while its number of rows is not affected by this variable.

\subsection{Unknown time receipts: a switched system viewpoint}

In the case where $N_{i}$ is unknown, a sufficient stability condition can be derived from [17, Th.6] by considering the time-varying controller (9b) instead of (9a).

\section{Lemma 4}

The system $\Sigma_{c l}$ is stable if there exist $P_{i} \in \mathbb{S}_{+}^{n}$ for all $i \in \mathcal{I}$ such that

$$
\Psi_{i}^{T} P_{j} \Psi_{i}-P_{i} \prec 0
$$

holds for all $(i, j) \in \mathcal{I}^{2}$.

Proof

See the proof of $[17$, Th.6] with obvious adaptation.

In view of [23, Th.1], the condition (21) can be interpreted as the constraint for the poly-quadratic Lyapunov functional

$$
V\left(\bar{x}_{n_{p}}, p\right)=\bar{x}_{n_{p}}^{T} P_{N_{p}} \bar{x}_{n_{p}}
$$

to be strictly decreasing along the trajectory of the linear switched system

$$
\bar{x}_{n_{p+1}}=\Psi_{N_{p}} \bar{x}_{n_{p}}
$$

where $N_{p} \in \mathcal{I}$. As suggested by the notation, identity (23) characterizes the dynamics of model (12) from $\bar{x}_{n_{p}}$ to $\bar{x}_{n_{p+1}}$, which appears to be a generalization of (17). In this context, $d_{k}$ is governed by $d_{k+1}=\left\lceil d_{k}+1\right\rceil_{N_{p}}$ for all $k \in\left[n_{p}, n_{p+1}\right)$. The dynamics of $d_{k}$ is periodic with a period which is randomly selected within the finite set $\mathcal{I}$. This is the motivation for qualifying such dynamics as "switched periodic" (and for this model (12) to inherit from this denomination). 


\section{Remark}

In stack contrast with Lemma 2, stability condition of Lemma 4 is merely sufficient since it is false, in general, that if $\Sigma_{c l}$ is stable then (21) holds. In view of the previous discussion, we conjecture that the lack of necessity originates from the fact that the existence of the strictly decreasing Lyapunov functional (22) cannot be ensured for all stable linear switched system of the form (23) (see [24]).

For the sake of completeness, the straightforward generalization of [17, Th.11] for the timevarying controller (9b) is now provided as a solution to the corresponding stabilization problem.

\section{Lemma 5}

The controller (9b) stabilizes the closed-loop model $\Sigma_{c l}$ if there exist (i) $W_{i} \in \mathbb{S}_{+}^{n}$ for all $i \in \mathcal{I}$, (ii) $Y_{k} \in \mathbb{R}^{m \times n}$ for all $k \in\left\{0, \ldots, N_{\max }-1\right\}$ and (iii) $G \in \mathbb{R}^{n \times n}$ such that

$$
\left[\begin{array}{cc}
-G-G^{T}+W_{i} & \star \\
A^{i} G+\sum_{k=0}^{i-1} A^{i-1-k} B Y_{k} & -W_{j}
\end{array}\right] \prec 0
$$

holds for all $(i, j) \in \mathcal{I}^{2}$. The gains of the control law are obtained by solving (19) with $W=G$ for all $k \in\left\{0, \ldots, N_{\max }-1\right\}$.

\section{Proof}

Substituting $Y_{i}$ by $K_{i} G$ in (24) followed by pre/post multiplication by $\left[\begin{array}{ll}\Psi_{i} & \mathbf{1}_{n}\end{array}\right]$ gives $\Psi_{i} W_{i} \Psi_{i}^{T}-$ $W_{j} \prec 0$. Using Schur complements, this inequality is equivalent to (21) with $P_{i}=W_{i}^{-1}$ and $P_{j}=$

$W_{j}^{-1}$, which in turn proves stability by Lemma 4 . Also note that (24) implies $-G-G^{T}+W_{i} \prec 0$, so $G$ is invertible since $W_{i} \succ 0$.

\section{Remark}

It is worth mentioning that all controllers ( $9 b$ ) which are proved to be stabilizing by Lemma 4 cannot be parametrized via Lemma 5. This is due to the fact that conservatism has been introduced from Lemma 4 to Lemma 5. To see it, first note that (21) holds if and only if there exist $G_{i} \in \mathbb{R}^{n \times n}$ for all $i \in \mathcal{I}$ satisfying

$$
\left[\begin{array}{cc}
-G_{i}-G_{i}^{T}+W_{i} & \star \\
\Psi_{i} G_{i} & -W_{j}
\end{array}\right] \prec 0, \forall(i, j) \in \mathcal{I}^{2}
$$

Indeed, (i) pre/post multiplication of (25) by $\left[\begin{array}{ll}\Psi_{i} & \mathbf{1}_{n}\end{array}\right]$ gives (21) for all $G_{i}$ and (ii) when $G_{i}=W_{i}$, (21) is proved to be equivalent to (25) by a Schur complement. Then, remarking that (25) is nothing but (24) with $G_{i}=G$ for all $i \in \mathcal{I}$, it follows that the conservatism from Lemma 4 to Lemma 5 originates from the constraint that a unique $G_{i}=G$ is involved in (25).

\section{THE FULL PROBLEM}

From now, the full problem is addressed so that both $\theta \in \Theta_{L}$ and $N_{p} \in \mathcal{I}=\left\{1, \ldots, N_{\text {max }}\right\}$ are supposed to be unknown. 


\subsection{Main results}

Immediate extension of Lemma 4 in this context reads: If for all $i \in \mathcal{I}$ and for all $\theta \in \Theta_{L}$, there exists $P_{i}(\theta) \in \mathbb{S}_{+}^{n}$ such that

$$
\Psi_{i}^{T}(\theta) P_{j}(\theta) \Psi_{i}(\theta)-P_{i}(\theta) \prec 0
$$

holds for all $\theta \in \Theta$ and for all $(i, j) \in \mathcal{I}^{2}$, then closed-loop system $\Sigma_{c l}(\theta)$ is robustly stable. Those matrix inequalities need to be satisfied on the continuous set $\Theta_{L}$ parametrized by $\theta$. Since $\Psi_{i}(\theta)$ is polynomial with respect to $\theta$ with degree $i$ (see (5) and (15)), it seems hard to design a tractable procedure which certifies that (26) is satisfied for all $\theta \in \Theta$. Although some directions relying on Sum Of Square (SOS) techniques are suggested in [25], the involved computational burden is expected to be prohibiting. For this reason, we seek to another procedure which aims exploiting the structure of $\Sigma_{c l}(\theta)$ by making use of the following matrices

$$
\begin{gathered}
\mathcal{N}_{i}:=\left[\begin{array}{cccccc}
A+B K_{0} & -\mathbf{1}_{n} & \mathbf{0} & \ldots & \ldots & \mathbf{0} \\
B K_{1} & A & -\mathbf{1}_{n} & \mathbf{0} & & \vdots \\
B K_{2} & \mathbf{0} & \ddots & \ddots & \ddots & \vdots \\
\vdots & \vdots & \ddots & A & -\mathbf{1}_{n} & \mathbf{0} \\
B K_{i-1} & \mathbf{0} & \ldots & \mathbf{0} & A & -\mathbf{1}_{n}
\end{array}\right], \\
\mathcal{N}_{i}:=\left[\begin{array}{ccccc}
A & \mathbf{0} & \ldots & \mathbf{0} & B K_{i-1} \\
-\mathbf{1}_{n} & A & \ddots & \vdots & \vdots \\
\mathbf{0} & \ddots & \ddots & \mathbf{0} & B K_{2} \\
\vdots & \ddots & -\mathbf{1}_{n} & A & B K_{1} \\
\vdots & & \mathbf{0} & -\mathbf{1}_{n} & A+B K_{0} \\
\mathbf{0} & \ldots & \ldots & \mathbf{0} & -\mathbf{1}_{n}
\end{array}\right],
\end{gathered}
$$

which should be regarded as a particular case of the ones introduced in [26, 19].

\section{Theorem 1 (Robust analysis)}

The polytopic model $\Sigma_{c l}(\theta)$ is robustly stable if one (at least) of the following conditions is satisfied:

- There exist matrices $P_{i}^{[q]} \in \mathbb{S}_{+}^{n}$ for all $(i, q) \in \mathcal{I} \times\{1, \cdots, L\}$ such that for all $(i, j) \in \mathcal{I}^{2}$ there exist $\mathcal{F}_{i, j} \in \mathbb{R}^{i n \times(i+1) n}$ satisfying

$$
\mathcal{X}_{i}\left(P_{i}^{[q]}, P_{j}^{[q]}\right)+\operatorname{He}\left\{\mathcal{F}_{i, j}^{T} \mathcal{N}_{i}^{[q]}\right\} \prec 0
$$

for all $q \in\{1, \cdots, L\}$.

- There exist matrices $W_{i}^{[q]} \in \mathbb{S}_{+}^{n}$ for all $(i, q) \in \mathcal{I} \times\{1, \cdots, L\}$ such that for all $(i, j) \in \mathcal{I}^{2}$ there exist $\mathcal{F}_{i, j} \in \mathbb{R}^{i n \times(i+1) n}$ satisfying

$$
\mathcal{X}_{i}\left(W_{j}^{[q]}, W_{i}^{[q]}\right)+\operatorname{He}\left\{\breve{\mathcal{N}}_{i}^{[q]} \mathcal{F}_{i, j}\right\} \prec 0
$$

for all $q \in\{1, \cdots, L\}$. 
The operator $\mathcal{X}_{i}$ is given by

$$
\mathcal{X}_{i}\left(P_{1}, P_{2}\right):=\left[\begin{array}{ccc}
-P_{1} & \mathbf{0} & \mathbf{0} \\
\mathbf{0} & \mathbf{0}_{(i-1) n} & \mathbf{0} \\
\mathbf{0} & \mathbf{0} & P_{2}
\end{array}\right]
$$

and matrices $\mathcal{N}_{i}^{[q]}$ and $\breve{\mathcal{N}}_{i}^{[q]}$ are readily defined from (27) and (28) by simply replacing $A$ and $B$ by $A^{[q]}$ and $B^{[q]}$ respectively.

Proof

Convex combinations of (29) for every $q \in\{1, \cdots, L\}$ lead to

$$
\mathcal{X}_{i}\left(P_{i}(\theta), P_{j}(\theta)\right)+\operatorname{He}\left\{\mathcal{F}_{i, j}^{T} \mathcal{N}_{i}(\theta)\right\} \prec 0
$$

with polytopic positive definite matrices $P_{i}(\theta)$ and $P_{j}(\theta)$ and where $\mathcal{N}_{i}(\theta)$ follows from the definition (27) by substituting $A$ and $B$ by $A(\theta)$ and $B(\theta)$ respectively. Then remarking that

$$
\mathcal{N}_{i}^{T \perp}=\left[\begin{array}{lllll}
\mathbf{1}_{n} & \Psi_{1}^{T} & \Psi_{2}^{T} & \ldots & \Psi_{i}^{T}
\end{array}\right]^{T},
$$

Lemma 1 ensures that (32) implies (26) as it holds

$$
\mathcal{N}_{i}^{T \perp} \mathcal{X}_{i}\left(P_{i}, P_{j}\right) \mathcal{N}_{i}^{T \perp T}=\Psi_{i}^{T} P_{j} \Psi_{i}-P_{i} .
$$

Since (26) holds for all $(i, j) \in \mathcal{I}^{2}$ and for all $\theta \in \Theta$, then $\Sigma_{c l}(\theta)$ is robustly stable. The same reasoning can be used when considering (30) instead of (29) by remarking that

$$
\breve{\mathcal{N}}_{i}^{\perp}=\left[\begin{array}{lllll}
\mathbf{1}_{n} & A & \ldots & A^{i-1} & \Psi_{i}
\end{array}\right]
$$

holds, and that

$$
\breve{\mathcal{N}}_{i}^{\perp} \mathcal{X}_{i}\left(P_{i}, P_{j}\right) \breve{\mathcal{N}}_{i}^{\perp T}=\Psi_{i} W_{i} \Psi_{i}^{T}-W_{j}
$$

is negative definite if and only if (21) holds with $P_{j}=W_{j}^{-1} \succ 0$ and $P_{i}=W_{i}^{-1} \succ 0$ by virtue of Schur complements.

Remark

Note that, in general, the two stability conditions provided by Theorem 1 are not equivalent. Section 5 provides numerical evidences of this fact.

Remark

An alternative strategy could be used to derive a robust tractable stability condition by relying on [14]. Indeed, this paper is devoted to stability analysis of systems of the form

$$
x_{k+1}=A(\theta) x_{k}+A_{d}(\theta) x_{k-d_{k}}
$$

which is close to (11) which governs dynamics of $\Sigma_{c l}(\theta)$. However, related conditions are expected to be more conservative since arbitrary dynamics is considered for $d_{k}$ in [14], i.e. $d_{k}$ can take arbitrary values in $\mathcal{I}$, whereas in this paper it is assumed that $d_{k}$ satisfies (8). 
The corresponding stabilization problem consists in computing the controller gains of (9b) such that closed-loop robust stability is ensured for all $N_{i} \in \mathcal{I}$ and for all $\theta \in \Theta$. This problem is tackled by considering those gains as decision variables of the optimization problems defined in Theorem 1 . Yet, this makes the matrix inequalities (29) and (30) bilinear due to the direct multiplication of $\mathcal{F}_{i, j}$ with $\mathcal{N}_{i}$ and $\breve{\mathcal{N}}_{i}$ respectively. To circumvent this issue, we focus on (30) and impose the last $n$ lines of every matrices $\mathcal{F}_{i, j}$ to be equal to $\left[\begin{array}{ll}\mathbf{0}_{n \times i n} & G\end{array}\right]$ so that $G \in \mathbb{R}^{n \times n}$ is a common matrix shared by every $\mathcal{F}_{i, j}$. Indeed, in such a case, it is possible to implement the change of variable $Y_{k}=K_{k} G$ to recover a linear dependency of (30) with respect to the variables of the new optimization problem. This manipulation preserves sufficiency since robust stability of the closed-loop model is ensured by the existence of $\mathcal{F}_{i, j}$ satisfying (30) irrespectively to the structure of this matrix. This gives rise to the following SDP.

\section{Theorem 2}

The controller (9b) stabilizes the polytopic closed-loop system $\Sigma_{c l}(\theta)$ if there exist (i) $W_{i}^{[q]} \in \mathbb{S}_{+}^{n}$ for all $(i, q) \in \mathcal{I} \times\{1, \cdots, L\}$, (ii) $Y_{k} \in \mathbb{R}^{m \times n}$ for all $k \in\left\{0 \ldots, N_{\max }-1\right\}$ and (iii) $G \in \mathbb{R}^{n \times n}$ such that for all $(i, j) \in \mathcal{I}^{2}$ there exists $\overline{\mathcal{F}}_{i, j} \in \mathbb{R}^{(i-1) n \times(i+1) n}$ verifying

$$
\begin{aligned}
& \mathcal{X}_{i}\left(W_{j}^{[q]}, W_{i}^{[q]}\right)+ \\
& \left.\operatorname{He}\left\{\left[\begin{array}{cccc}
A^{[q]} & \mathbf{0} & \cdots & \mathbf{0} \\
-\mathbf{1} & A^{[q]} & \ddots & \vdots \\
\mathbf{0} & \ddots & \ddots & \mathbf{0} \\
\vdots & \ddots & -\mathbf{1} & A^{[q]} \\
\mathbf{0} & \cdots & \mathbf{0} & -\mathbf{1}
\end{array}\right]\left[\begin{array}{cc}
\overline{\mathcal{F}}_{i, j} & \\
\mathbf{0}_{n \times i n} & G
\end{array}\right]+\left[\begin{array}{c}
{\left[B^{[q]} Y_{i-1}\right.} \\
\vdots \\
B^{[q]} Y_{0} \\
\mathbf{0}_{n}
\end{array}\right]\right]\right\} \prec 0
\end{aligned}
$$

for all $q \in\{1, \cdots, L\}$. The gains of the control law are obtained by solving (19) with $W=G$ for all $i \in\left\{0, \ldots, N_{\max }-1\right\}$.

\section{Proof}

Substituting $Y_{k}$ by $K_{k} G$ in (38) gives (30) with $F_{i, j}=\left[\begin{array}{c}\overline{\mathcal{F}}_{i, j} \\ {\left[\begin{array}{cc}\mathbf{0} & G\end{array}\right]}\end{array}\right]$, which proves robust stability by Lemma 1. Also note that if (38) is satisfied, then the matrix $G$ is invertible. Indeed, by focusing on the bottom right-hand side block of size $n \times n$ of (38), it comes that $W_{i}^{[q]}-G-G^{T} \prec 0$ which together with $W_{i}^{[q]} \succ 0$ proves that $\operatorname{det}(G) \neq 0$.

\section{Remark}

In order to derive a constant controller of the form (9a), it suffices to substitute in Theorem 2 a single matrix $Y$ to every $Y_{i}$. The controller gain can be then recovered by computing $K=Y G^{-1}$. Although more conservative (see Section 5), this manipulation might be desirable when implementation constraints prevent the designer from using the time-varying control law (9b).

\subsection{Discussion about the conservatism of the proposed approach}

This subsection aims at providing more details about the conservatism associated with Theorems 1 and 2 . 
4.2.1. Conditions associated with Theorems 1 and 2 in the nominal case: The conditions proposed in Theorems 1 and 2 are merely sufficient. This suggests that they may fail to provide stability certificates although closed-loop system $\Sigma_{c l}(\theta)$ is robustly stable. Nevertheless, it can be proved that at least in the nominal case, i.e. when $\theta$ is known which corresponds to $L=1$, if Lemma 4 (Lemma 5) successfully proves stability, then conditions associated with Theorem 1 (Theorem 2) are satisfied. In other words, when $L=1$, Theorems 1 and 2 are equivalent to Lemma 4 and 5 respectively (recall that conditions of Theorems 1 and 2 imply that of Lemma 4 and 5 respectively). This is a straightforward consequence of the two following propositions.

\section{Proposition 1}

For given $P_{i} \in \mathbb{S}_{+}^{n}$ and $P_{j} \in \mathbb{S}_{+}^{n}$, the following statements are equivalent to (21):

1. There exists $\mathcal{F}_{i, j} \in \mathbb{R}^{i n \times(i+1) n}$ verifying (29) with $P_{i}^{[q]}=P_{i}, P_{j}^{[q]}=P_{j}$ and $\mathcal{N}_{i}^{[q]}=\mathcal{N}_{i}$.

2. There exists $\mathcal{F}_{i, j} \in \mathbb{R}^{i n \times(i+1) n}$ verifying (30) with $W_{i}^{[q]}=P_{i}^{-1}, W_{j}^{[q]}=P_{j}^{-1}$ and $\breve{\mathcal{N}}_{i}^{[q]}=\breve{\mathcal{N}}_{i}$.

Proof

The fact that (21) is equivalent to " 1. " readily follows from the use of Lemma 1 together with the identity (34) on (21). To prove equivalence between (21) and "2.", it suffices to rewrite (21) by means of Schur complements and then to use (36) together with Lemma 1.

Proposition 2

For given $W_{i} \in \mathbb{S}_{+}^{n}, W_{j} \in \mathbb{S}_{+}^{n}, G \in \mathbb{R}^{n \times n}$ and $Y_{k} \in \mathbb{R}^{m \times n}$ for all $k \in\left\{0, \ldots, N_{\max }-1\right\}$, (24) holds if and only if there exists $\overline{\mathcal{F}}_{i, j} \in \mathbb{R}^{(i-1) n \times(i+1) n}$ verifying (38) with $W_{i}^{[q]}=W_{i}$ and $W_{j}^{[q]}=W_{j}$.

Proof

Let us first introduce

$$
\Pi:=\left[\begin{array}{cccccc}
A^{T} & -\mathbf{1} & \mathbf{0} & \cdots & \cdots & \mathbf{0} \\
\mathbf{0} & \ddots & \ddots & \ddots & & \vdots \\
\vdots & \ddots & \ddots & \ddots & \ddots & \vdots \\
\mathbf{0} & \cdots & \mathbf{0} & A^{T} & -\mathbf{1} & \mathbf{0}
\end{array}\right] \in \mathbb{R}^{(i-1) n \times(i+1) n} .
$$

Remark that

$$
\Pi^{T \perp}=\left[\begin{array}{ccccc}
\mathbf{0} & \mathbf{0} & \ldots & \mathbf{0} & \mathbf{1} \\
\mathbf{1} & A & \ldots & A^{i-1} & \mathbf{0}
\end{array}\right]
$$

Then,

$$
\begin{array}{r}
{\left[\begin{array}{cc}
-G-G^{T}+W_{i} & \star \\
A^{i} G+\sum_{j=0}^{i-1} A^{i-1-j} B Y_{j} & -W_{j}
\end{array}\right]=} \\
\left.\Pi^{T \perp}\left(\left[\begin{array}{c}
B Y_{i-1} \\
\vdots \\
\mathbf{0} \\
\\
B Y_{0}+A G \\
-G
\end{array}\right]\right\} W_{j}, W_{i}\right)+\operatorname{He}\left\{\Pi^{T \perp T}\right.
\end{array}
$$


proves, by Lemma 1, that (24) holds if and only if there exists $\overline{\mathcal{F}}_{i, j} \in \mathbb{R}^{(i-1) n \times(i+1) n}$ such that

$$
\mathcal{X}_{i}\left(W_{j}, W_{i}\right)+\operatorname{He}\left\{\Pi^{T} \overline{\mathcal{F}}_{i, j}+\left[\begin{array}{c} 
\\
B Y_{i-1} \\
\vdots \\
B Y_{0}+A G \\
-G
\end{array}\right]\right\} \prec 0
$$

which is nothing but (38) with $W_{i}^{[q]}=W_{i}$ and $W_{j}^{[q]}=W_{j}$.

4.2.2. Source of conservatism in Theorem 1 : Let us now give more insights about the origin of the conservatism associated with Theorem 1 and 2. To remain concise, we focus on the condition associated with (29). Similar conclusions can be easily drawn if (30) and (38) are considered.

On the first hand, it comes from (34) that (26) is verified if and only if there exists $\mathcal{F}_{i, j}(\theta)$ such that

$$
\mathcal{X}_{i}\left(P_{i}(\theta), P_{j}(\theta)\right)+\operatorname{He}\left\{\mathcal{F}_{i, j}^{T}(\theta) \mathcal{N}_{i}(\theta)\right\} \prec 0,
$$

by virtue of Lemma 1 . At this point, arbitrary dependency with respect to $\theta$ should be considered for both $P_{i}(\theta), P_{j}(\theta)$ and $\mathcal{F}_{i, j}(\theta)$. On the other hand, as mentioned in the proof of Theorem 1, convex combination of (29) leads to (32) with polytopic matrices $P_{i}(\theta)$ and $P_{j}(\theta)$ which is nothing but (43) with constant $\mathcal{F}_{i, j}(\theta)$, that is independent of $\theta$. This suggests that if there exist a polytopic $P_{i}(\theta)$ and a constant $\mathcal{F}_{i, j}(\theta)$ satisfying (43) then the analysis condition related to this inequality can be cast into the SDP associated with (29) in Theorem 1. At first glance, it seems that the conservatism associated with (29) originates from those restrictions on the classes in which $P_{j}(\theta)$ and $\mathcal{F}_{i, j}(\theta)$ belong to, namely polytopic for $P_{i}(\theta)$ and constant for $\mathcal{F}_{i, j}(\theta)$. However, the next proposition suggests a different interpretation.

\section{Proposition 3}

The two following facts hold:

1. If there exist $P_{i}(\theta) \succ 0$ and $P_{j}(\theta) \succ 0$ such that (26) holds, then there exists $\mathcal{F}_{i, j}(\theta)$ verifying (43) and which is linear with respect to $\theta$.

2. If (43) holds with constant matrix $\mathcal{F}_{i, j}$ and $P_{i}(\theta) \succ 0$ and $P_{j}(\theta) \succ 0$ with arbitrary dependency with respect to $\theta$, then there exist polytopic matrices $\hat{P}_{j}(\theta)$ and $\hat{P}_{i}(\theta)$ satisfying (43) with the same $\mathcal{F}_{i, j}$.

\section{Proof}

With (34) and Lemma 1, it follows that if (26) holds then there exists $\rho \in \mathbb{R}$ such that $\mathcal{X}_{i}\left(P_{i}(\theta), P_{j}(\theta)\right)+\rho \mathcal{N}_{i}^{T}(\theta) \mathcal{N}_{i}(\theta) \prec 0$ which is nothing but (43) with $\mathcal{F}_{i, j}(\theta)=\rho / 2 \quad \mathcal{N}_{i}(\theta)$. Linearity with respect to $\theta$ comes from (27). This clearly proves the first fact.

As far as the second fact is concerned, observe that if (43) holds for all $\theta \in \Theta_{L}$ with constant matrices $\mathcal{F}_{i, j}$, then (43) holds in particular for all vertices of the polytope $\Theta_{L}$, i.e. for all $\theta=e_{q}$ where $e_{q}$ corresponds to the $q$-th unit vector of dimension $L$. Convex combination on $q$ of the resulting inequalities leads to (43) with polytopic matrices $\hat{P}_{i}(\theta)=\sum_{q=1}^{L} \theta_{q} P_{i}\left(e_{q}\right)$ and $\hat{P}_{j}(\theta)$ defined similarly. 
Thus, we conclude that the only source of conservatism (yet not negligible) from condition associated to (26) to the first stability condition of Theorem 1 comes from the constraint on $\mathcal{F}_{i, j}(\theta)$ to be independent of $\theta$ while, in general, this matrix is linear with respect to $\theta$. Hence, considering polytopic matrices $P_{i}(\theta)$ is not restrictive in this context.

\section{NUMERICAL RESULTS}

In this section, several examples are used to illustrate the effectiveness of the proposed results.

\section{Example 1}

Consider the following numerical system borrowed from [8, Ex. 2]:

$$
x_{k+1}=\left(A_{0}+\alpha A_{\alpha}\right) x_{k}+A_{d} x_{k-d_{k}}
$$

with

$$
A_{0}=\left[\begin{array}{cc}
0.8 & 0 \\
0 & 0.9
\end{array}\right], A_{\alpha}=\left[\begin{array}{ll}
1 & 0 \\
0 & 0
\end{array}\right], A_{d}=\left[\begin{array}{cc}
-0.1 & 0 \\
-0.1 & -0.1
\end{array}\right]
$$

and where $\alpha$ is an unknown parameter which has constant value satisfying $|\alpha| \leq \alpha_{M}$. It is considered that $d_{k}$ is governed by (8) and such that $1 \leq n_{p+1}-n_{p} \leq N_{\max }=5$ for all $p \in \mathbb{N}$. We are interested in determining the upper value $\alpha_{M}$ such that the above system is robustly stable.

This analysis problem can be recast into the framework considered in this paper by regarding (44) as the closed-loop model (10) with $B(\theta)=A_{d}, K_{d_{k}}=\mathbf{1}_{2}$ for all $d_{k}$ and $A(\theta)=\theta_{1}\left(A_{0}-\alpha_{M} A_{\alpha}\right)+$ $\theta_{2}\left(A_{0}+\alpha_{M} A_{\alpha}\right)$ where $\theta=\left(\theta_{1}, \theta_{2}\right) \in \Theta_{2}$. Lower bound of $\alpha_{M}$ is computed by means of robust stability conditions of Theorem 1: Both conditions (29) and (30) ensure that $\alpha_{M} \geq 0.30$.

Results presented in [9] and [8] have been derived in a different context as $d_{k}$ is allowed to take arbitrary value within $\{3, \ldots, 5\}$ and $\alpha$ is time-varying. In such a case, it is proved that $\alpha_{M} \geq 0.16$ and $\alpha_{M} \geq 0.24$ in [9] and [8] respectively, which is in both cases smaller than $\alpha_{M} \geq 0.30$ obtained via (29). This suggests that Theorem 1 might be preferred whenever $\alpha$ is constant and $d_{k}$ is governed by (8) as, in this context, its condition can be less conservative than that of [9] and [8].

\section{Example 2}

In the previous example, the two conditions of Theorem 1 lead to the same result. However, in general, they are not equivalent. This fact is highlighted in the following counterexample:

$$
x_{k+1}=\left(A_{0}+\alpha A_{\alpha}\right) x_{k}+B K_{d_{k}} x_{k-d_{k}}
$$

with

$$
\begin{array}{r}
A_{0}=\left[\begin{array}{ccc}
0.70 & -0.90 & -0.20 \\
0 & -0.40 & -0.50 \\
0.80 & 0.80 & -0.60
\end{array}\right], A_{\alpha}=\left[\begin{array}{ccc}
0.20 & -0.10 & 0.20 \\
0.30 & -0.10 & -0.40 \\
-0.20 & 0.20 & -0.10
\end{array}\right], B=\left[\begin{array}{cc}
0.10 & 0 \\
-0.10 & 0.10 \\
0.10 & 0
\end{array}\right] \\
K_{0}=\left[\begin{array}{ccc}
-1.68 & 0.08 & -3.61 \\
-0.20 & 6.26 & 1.18
\end{array}\right], K_{1}=\left[\begin{array}{ccc}
-3.85 & 2.09 & 6.59 \\
1.39 & 5.67 & 1.17
\end{array}\right]
\end{array}
$$


Table I. Computational effort associated with Theorem 2 (Example 3)

\begin{tabular}{|c|c|c|c|}
\hline \multirow{2}{*}{$N_{\max }$} & \multirow{2}{*}{ Nb of rows } & \multicolumn{2}{|c|}{ Nb of var. } \\
\cline { 3 - 4 } & & $K$ & $K_{k}$ \\
\hline 2 & 48 & 42 & 44 \\
3 & 120 & 156 & 160 \\
4 & 240 & 446 & 452 \\
5 & 420 & 1036 & 1044 \\
6 & 672 & 2082 & 2092 \\
7 & 1008 & 3772 & 3784 \\
\hline
\end{tabular}

where $d_{k}$ verifies (8) and is such that $1 \leq n_{p+1}-n_{p} \leq N_{\max }=3$ for all $p \in \mathbb{N}$ and $\alpha$ is an unknown parameter which has constant value satisfying $|\alpha| \leq \alpha_{M}$. In such a case, different bounds of $\alpha_{M}$ are delivered by Theorem 1: (29) (respectively (30)) guaranties that $\alpha_{M} \geq 1.77$ (resp. $\alpha_{M} \geq 1.65$ ).

\section{Example 3}

Consider the following uncertain discrete-time system

$$
x_{k+1}=\left(A_{0}+\alpha A_{\alpha}\right) x_{k}+B u_{k}
$$

with

$$
A_{0}=\left[\begin{array}{cc}
0.3 & 0.6 \\
1.5 & 1
\end{array}\right], A_{\alpha}=\left[\begin{array}{ll}
0 & 0 \\
1 & 1
\end{array}\right], B=\left[\begin{array}{l}
1.3 \\
1.4
\end{array}\right]
$$

and where $\alpha$ is an unknown parameter which has constant value satisfying $0 \leq \alpha \leq \alpha_{M}$. Assume that information about the current state $x_{k}$ is delivered through a network subject to packet drop so that only $x_{n_{p}}$ can be used by the controller for all $k \in\left[n_{p}, n_{p+1}\right)$. For different value of $N_{\max }=$ $\max \left(n_{p+1}-n_{p}\right)$, our purpose is to compute time-invariant controller (9a) and time-varying control law (9b) with $K_{k}=K_{d_{k}}$ which maximizes the closed-loop stability domain parametrized by $\alpha_{M}$.

First note that (49) is nothing but (4) and (5) with $A^{[1]}=A_{0}, A^{[2]}=A_{0}+\alpha_{M} A_{\alpha}$ and $B^{[q]}=B$ for $q \in\{1,2\}$. Consequently, controller gains can be computed using Theorem 2 . The related computational effort is evaluated in terms of number of rows and variables and is recorded in Table I. The number of rows is independent of the kind of controller that is considered whereas $n m\left(N_{\max }-1\right)$ extra variables should be introduced whenever $K_{k}$ is retained. From Table I, it appears that the required computational effort is very similar for the two kind of controllers.

Nevertheless, very different results can be observed when computing the guaranteed lower bound of $\alpha_{M}$ for the resulting closed-loop systems. This value is evaluated by retaining the highest value obtained via the two conditions in Theorem 1. Results are depicted in Figure 3. It can be seen that the stability domain is significantly enlarged by considering the time-varying controller $K_{k}$. For instance, when $N_{\max }=7$, the guaranteed lower bound of $\alpha_{M}$ is improved by $926 \%$ when using $K_{k}$ instead of $K$.

Let us now consider the controller $K_{k}$ and the matrices $W_{i}^{[q]}$ arising from Theorem 2 for $N_{\max }=7$ and $\alpha_{M}=0.36$, which corresponds to the upper bound of the guaranteed stability domain. From the matrices $W_{i}^{[q]}$, we construct the functional

$$
V\left(x_{k}, \theta, p\right)=x_{k}^{T} W_{i=N_{p}}^{-1}(\theta) x_{k},
$$




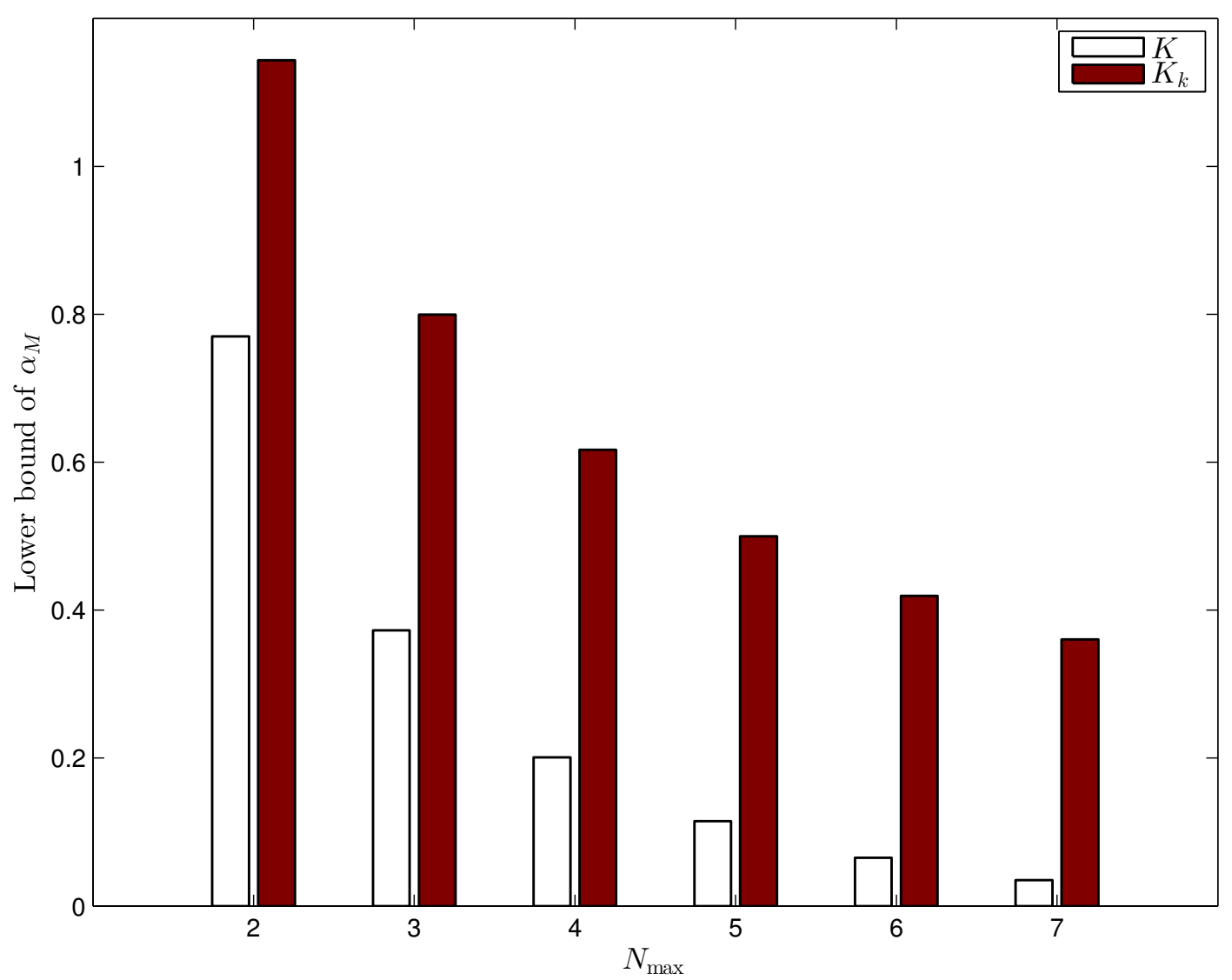

Figure 3. Guaranteed lower bound of $\alpha_{M}$ for time-invariant and time-varying controllers (Example 3)

where $W_{i}(\theta)$ is the polytopic matrix which vertices are $W_{i}^{[1,2]}$ and $p$ is such that the inequalities $n_{p} \leq k<n_{p+1}$ are satisfied. Our purpose is to show how $V\left(x_{k}, \theta, p\right)$ evolves when $x_{k}$ is the state vector of the closed-loop (49) with $K_{k}$. Indeed, this functional plays a keep role in the proof of closed-loop stability as Theorem 2 ensures that

$$
V\left(x_{n_{p+1}}, \theta, p+1\right)-V\left(x_{n_{p}}, \theta, p\right)<0
$$

for all $(\theta, p) \in \Theta_{2} \times \mathbb{N}$. Let us regularly sample the parametric space $\Theta_{2}$ so that $\theta_{1}$ takes value in $\{0,0.5,1\}$ which leads to 3 closed-loop models. Each of those systems are simulated for $x_{0}=(-0.8,0.6)$ and the following sequence $n_{i}$ :

$$
\left(n_{0}, \ldots, n_{9}\right)=(0,7,9,14,17,18,23,24,27,34)
$$

Chronograph of the values of $V\left(x_{k}, \theta, p\right)$ are depicted in Figure 4 for the above samples of $\Theta_{2}$. Dash-dot lines with marker "o" are samples of $V\left(x_{k}, \theta, p\right)$ for instant times $k=n_{p}$. Note that the lower subplot illustrates the packet-drop sequence so that 1 (resp. 0) indicates success (resp. fail) of the transmission.

It can be verified that the inequality (52) holds for all considered values of $\theta$ and $n_{p}$. This is a direct consequence of the fact that this functional is a suitable Lyapunov functional for the switched model 
(23). However, $V\left(x_{k}, \theta, p\right)$ is not necessarily decreasing for all $k$ verifying $n_{p} \leq k<n_{p+1}$. This can be observed in particular from the time interval $\left[n_{0}, n_{1}\right]=[0,7]$ where none of the functionals $V\left(x_{k}, \theta, p\right)$ is always decreasing, even if $V\left(x_{7}, \theta, 7\right)<V\left(x_{0}, \theta, 0\right)$ holds for all samples of $\Theta_{2}$.
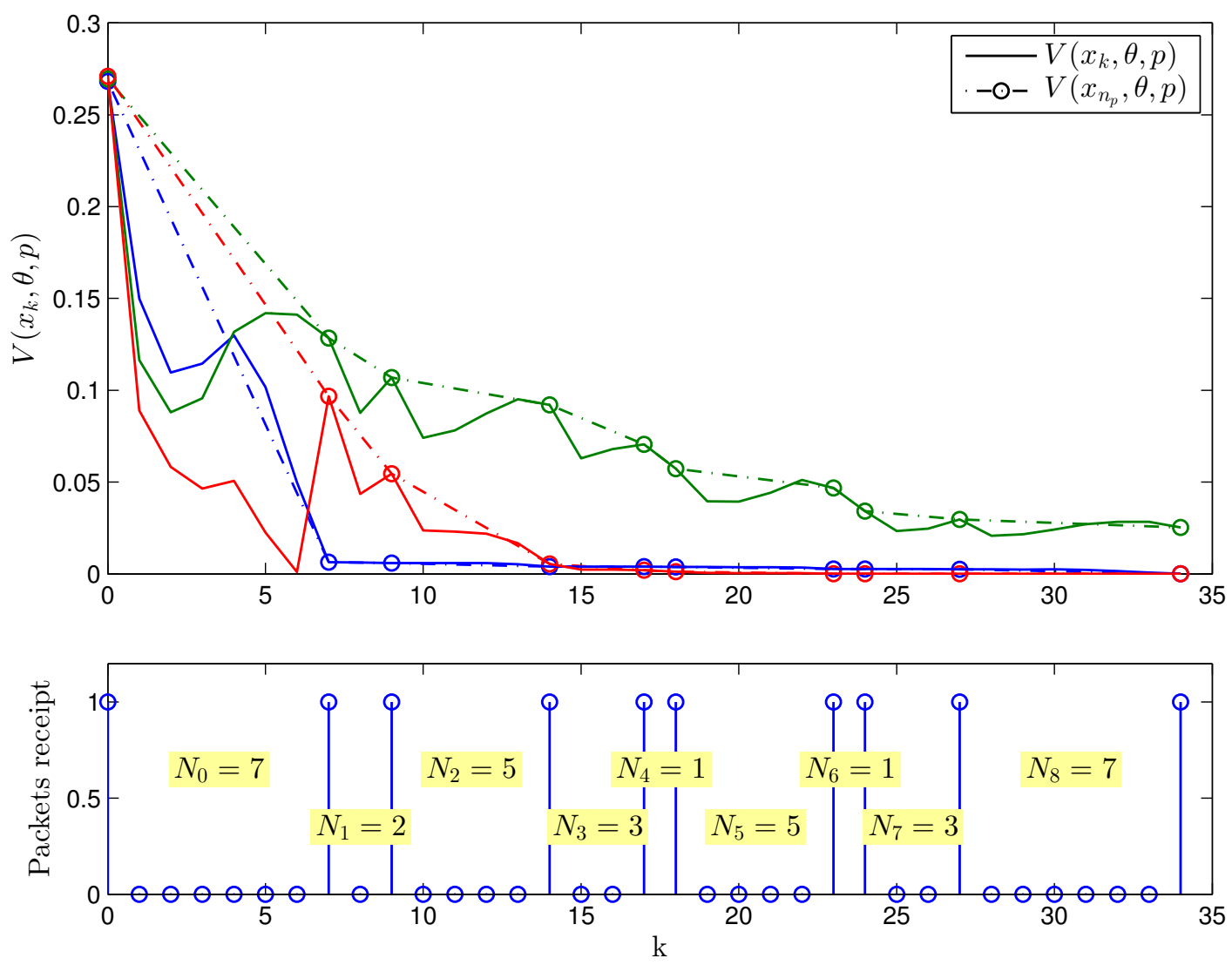

Figure 4. Chronograph of the functional $V\left(x_{k}, \theta, p\right)$ for samples of $\Theta_{2}$ (Example 3)

\section{CONCLUSIONS}

This paper presented a novel approach to assess stability and stabilization of discrete-time linear systems subject to parameters uncertainties and packet losses. The method is based on a reformulation of the problem into the framework of robust control. We have provided several stability conditions whose effectiveness is supported by numerical experiments. Future works would first consist in including some additional analysis to estimate the input/output performances, like in [27]. The corresponding output feedback stabilization problem will also be considered in the near future, possibly via a two-step design making use of an observer/predictor block. Another aspect comes from the fact that the proposed method requires a notable increase of the complexity of the LMI problem. Thus, further study is driven by the idea of reducing this complexity, but with a particular attention to the associated conservatism. 


\section{REFERENCES}

1. Zampieri S. A survey of recent results in Networked Control Systems. Proc. $17^{\text {th }}$ IFAC World Congress, Seoul, Korea, 2008.

2. Hespanha J, Naghshtabrizi P, Xu Y. A survey of recent results in networked control systems. Proceedings of the IEEE 2007; 95(1):138-162.

3. Fridman E, Seuret A, Richard JP. Robust sampled-data stabilization of linear systems: An input delay approach. Automatica 2004; 40(8):1141-1446.

4. Liu K, Fridman E. Discrete-time network-based control under scheduling and actuator constraints. International Journal of Robust and Nonlinear Control 2014; .

5. Fridman E, Shaked U. Delay-dependent $H_{\infty}$ control of uncertain discrete delay systems. European Journal of Control 2005; 11(1):29-37.

6. Fridman E, Shaked U. Stability and guaranteed cost control of uncertain discrete delay systems. International Journal of Control 2005; 78(4):235-246.

7. Jiang X, Han QL, Yu X. Stability criteria for linear discrete-time systems with interval-like time-varying delay. American Control Conference, 2005. Proceedings of the 2005, IEEE, 2005; 2817-2822.

8. Gao H, Chen T. New results on stability of discrete-time systems with time-varying state delay. Automatic Control, IEEE Transactions on 2007; 52(2):328-334.

9. Gao H, Lam J, Wang C, Wang Y. Delay-dependent output-feedback stabilisation of discrete-time systems with time-varying state delay. IEEE Proceedings-Control Theory and Applications 2004; 151(6):691-698.

10. Kao CY. On stability of discrete-time LTI systems with varying time delays. Automatic Control, IEEE Transactions on 2012; 57(5):1243-1248.

11. Sun XM, Liu GP, Rees D, Wang W. Delay-dependent stability for discrete systems with large delay sequence based on switching techniques. Automatica 2008; 44(11):2902-2908.

12. Zhang WA, Yu L. Modelling and control of networked control systems with both network-induced delay and packetdropout. Automatica 2008; 44(12):3206-3210.

13. Hespanha JP, Morse S. Stability of switched systems with average dwell-time. Decision and Control, 1999. Proceedings of the 38th IEEE Conference on, vol. 3, IEEE, 1999; 2655-2660.

14. Hetel L, Daafouz J, Iung C. Equivalence between the Lyapunov-Krasovskii functionals approach for discrete delay systems and that of the stability conditions for switched systems. Nonlinear Analysis: Hybrid Systems 2008; 2(3):697 - 705. Special Issue Section: Analysis and Design of Hybrid Systems Analysis and Design of Hybrid Systems.

15. Xiao L, Hassibi A, How JP. Control with random communication delays via a discrete-time jump system approach. American Control Conference, 2000. Proceedings of the 2000, vol. 3, IEEE, 2000; 2199-2204.

16. Li H, Chow Y, Sun Z. Optimal stabilizing gain selection for networked control systems with time delays and packet losses. Control Systems Technology, IEEE Transactions on 2009; 17(5):1154-1162.

17. Xiong J, Lam J. Stabilization of linear systems over networks with bounded packet loss. Automatica 2007; 43(1):80 $-87$.

18. Ebihara Y, Peaucelle D, Arzelier D. Periodically time-varying memory state-feedback controller synthesis for discrete-time linear systems. Automatica 2011; 47(1):14 - 25.

19. Trégouët JF, Peaucelle D, Arzelier D, Ebihara Y. Periodic memory state-feedback controller: New formulation, analysis and design results. Automatic Control, IEEE Transactions on 2013; 58(8):1986 - 2000.

20. Seuret A. A novel stability analysis of linear systems under asynchronous samplings. Automatica 2012; 48(1):177182.

21. Skelton RE, Iwasaki T, Grigoriadis DE. A Unified Algebraic Approach to Linear Control Design. Taylor \& Francis Ltd: London, UK, 1997.

22. Bittanti S, Colaneri P. Periodic Systems: Filtering and Control. 1st edn., Springer Publishing Company, Incorporated, 2008.

23. Mignone D, Ferrari-Trecate G, Morari M. Stability and stabilization of piecewise affine and hybrid systems: An LMI approach. Decision and Control, 2000. Proceedings of the 39th IEEE Conference on, vol. 1, IEEE, 2000; 504-509.

24. Lin H, Antsaklis PJ. Stability and stabilizability of switched linear systems: a survey of recent results. Automatic control, IEEE Transactions on 2009; 54(2):308-322.

25. Scherer CW. Relaxations for robust linear matrix inequality problems with verifications for exactness. SIAM J. Matrix Anal. Appl. 2005; 27(2):365-395.

26. Trégouët JF, Ebihara Y, Arzelier D, Peaucelle D, Pittet C, Falcoz A. Robust stability of periodic systems with memory: New formulations, analysis and design results. Proceedings of the 7th IFAC Symposium on Robust Control 
Design, vol. 7, Aalborg, Denmark, 2012; 684-689.

27. Gao H, Chen T. Hinf estimation for uncertain systems with limited communication capacity. Automatic Control, IEEE Transactions on 2007; 52(11):2070-2084. 